\title{
Análisis del proceso de recuperación de la batería de alta tensión del vehículo Hyundai Sonata híbrido
}

\section{Analysis of the recovery process of the high tension battery of the Hyundai Sonata hybrid vehicle}

\author{
Daniela Alexandra Jerez Mayorga \\ Universidad Internacional del Ecuador, Ecuador \\ Edwin Giovanny Puente Moromenacho \\ Universidad Internacional del Ecuador, Ecuador
}

Autor para correspondencia: djerez@uide.edu.ec; epuente@uide.edu.ec

Fecha de recepción: 15 de agosto de 2018 - Fecha de aceptación: 15 octubre de 2018

Resumen: En este trabajo se analiza el proceso de recuperación de baterías del vehículo Hyundai Sonata Híbrido, mediante la revisión diagnóstica con distintos bancos de trabajo y comprobadores. También se da a conocer el nuevo banco de pruebas para baterías híbridas Charger Research como un equipo de análisis de las celdas del pack de baterías para vehículos híbridos y eléctricos, el cual cubre las necesidades de revisión del sistema de alto voltaje entorno al paquete de baterías, analizando el funcionamiento celda por celda permitiendo su recuperación, proceso que hasta el día de hoy aún no se usa a gran escala en el país, generando una alternativa que permite alargar la vida útil de las baterías. Estas aplicaciones permiten dar un paso al avance en el área automotriz permitiendo generar nuevas oportunidades laborales, con más razón sabiendo que el porcentaje de ventas de vehículos híbridos en el país ha crecido considerablemente desde su aparición en el año 2009, y aunque los fabricantes de estos modelos hablan de garantías que cubren al adquirir un vehículo amigable al ecosistema, aún muchos propietarios se preocupan por no saber qué hacer o donde acudir cuando el sistema de alto voltaje de su vehículo comience a fallar.

Palabras Clave: híbrido; batería; recuperación; carga; ahorro

Abstract: In this paper, the battery recovery process of the Hyundai Sonata Hybrid vehicle is analyzed, through the diagnostic review with different workbenches and testers. The new battery test bench for Charger Research hybrid batteries is also announced as a battery pack analysis equipment for hybrid and electric vehicles, which covers the needs of reviewing the high voltage system around the battery pack, analyzing the operation cell by cell allowing its recovery, process that until today is still not used on a large scale in the country, generating an alternative that allows to extend the useful life of the batteries. These applications make it possible to take a step forward in the automotive area by generating new job opportunities, with more reason knowing that the percentage of sales of hybrid vehicles in the country has grown considerably since its appearance in 2009, and although the manufacturers of these Models talk about the guarantees they cover when purchasing a vehicle that is friendly to the ecosystem, yet many owners worry about not knowing what to do or where to turn when the high voltage system of their vehicle starts.

Key words: hybrid; battery; recovery; charge; saving 


\section{Introducción}

Las grandes cifras de contaminación mundial preocupan a las grandes empresas automovilísticas, quienes se ven obligadas a crear nuevas alternativas para la movilidad automovilística. Es así como en el mercado mundial existen algunas marcas que apuestan a la fabricación de autos con nuevas tecnologías, lanzando al mercado modelos como vehículos híbridos y eléctricos, Toyota, Kía, Chevrolet, Ford entre otras, son las casas que apuestan a estas nuevas soluciones ambientalistas.

La marca surcoreana Hyundai también se inclina por esta gama de vehículos y en el país introdujo el primer auto, se trata de un sedán cuatro puertas llamado Sonata Hybrid, en su versión 2014. Posee tecnología de batería de polímeros de litio de siguiente generación, "la primera batería para híbridos con garantía de por vida", según la marca. Gracias a esta tecnología recorre $70 \mathrm{~km}$ con un galón de combustible. Este sedán fue presentado por primera vez en el Salón del Automóvil de Nueva York, en el 2010. El primer mercado al que se introdujo fue el surcoreano, en ese mismo año.

Ecuador es el primer país latinoamericano al que llega este automotor. Según aseguró José Ávila, gerente de Marketing de la firma en Ecuador, comenta que para ese año tenían previsto importar unas 400 unidades de este modelo. (Revista Líderes - El Comercio, 2013)

En la tabla 1 se muestra el número de ventas de vehículos híbridos a nivel nacional desde el año 2010 al 2016, con un total de 11.552 unidades vendidas en todo el país, de las cuales 1832 unidades corresponden a la marca Hyundai desde su aparición en el marcado con modelos híbridos en el 2014, lo que representa aproximadamente el $15.9 \%$ de ventas, posicionándolo en el mercado como número uno a nivel nacional en los tres últimos años.

Tabla 1. Ventas nacionales de vehículos híbridos por marca (AEADE, 2017)

\begin{tabular}{|c|c|c|c|c|c|c|c|c|c|c|c|c|c|c|}
\hline \multicolumn{15}{|c|}{ VENTAS DE VEHÍCULOS HÍBRIDOS POR MARCA } \\
\hline \multicolumn{15}{|c|}{ En unidades / porcentaje de participación 2010 - 2016} \\
\hline MARCA & 2010 & $\%$ & 2011 & $\%$ & 2012 & $\%$ & 2013 & $\%$ & 2014 & $\%$ & 2015 & $\%$ & 2016 & $\%$ \\
\hline HYUNDAI & - & $0,00 \%$ & - & $0,00 \%$ & - & $0,00 \%$ & - & $0.00 \%$ & 470 & $52,69 \%$ & 717 & $78.88 \%$ & 645 & $65.09 \%$ \\
\hline TOYOTA & 1840 & $40,81 \%$ & 557 & $23,91 \%$ & 1352 & $96,57 \%$ & 417 & $80.04 \%$ & 304 & $34,08 \%$ & 155 & $17,05 \%$ & 218 & $22,00 \%$ \\
\hline $\mathrm{KIA}$ & - & $0,00 \%$ & - & $0,00 \%$ & - & $0.00 \%$ & - & $0.00 \%$ & 74 & $8.30 \%$ & 20 & $2,20 \%$ & 123 & $12,41 \%$ \\
\hline PORSCHE & 54 & $1,20 \%$ & 111 & $4,76 \%$ & 25 & $1.79 \%$ & 1 & $0.19 \%$ & 4 & $0.45 \%$ & 7 & $0,77 \%$ & 3 & $0.30 \%$ \\
\hline HONDA & - & $0.00 \%$ & - & $0.00 \%$ & - & $0.00 \%$ & $\cdot$ & $0.00 \%$ & 1 & $0.11 \%$ & 2 & $0,22 \%$ & 2 & $0.20 \%$ \\
\hline BMW & 154 & $3,42 \%$ & 82 & $3,52 \%$ & 5 & $0,36 \%$ & - & $0,00 \%$ & 23 & $2,58 \%$ & 3 & $0,33 \%$ & - & $0.00 \%$ \\
\hline AUDI & - & $0.00 \%$ & - & $0.00 \%$ & - & $0,00 \%$ & - & $0.00 \%$ & 7 & $0.78 \%$ & - & $0,00 \%$ & - & $0.00 \%$ \\
\hline FORD & 1056 & $23,42 \%$ & 1034 & $44,38 \%$ & 3 & $0,21 \%$ & 33 & $6,33 \%$ & 2 & $0.22 \%$ & 3 & $0.33 \%$ & - & $0.00 \%$ \\
\hline VOLKSWAGEN & - & $0.00 \%$ & - & $0.00 \%$ & - & $0.00 \%$ & - & $0.00 \%$ & 1 & $0.11 \%$ & - & $0.00 \%$ & - & $0.00 \%$ \\
\hline NISSAN & - & $0,00 \%$ & $\cdot$ & $0,00 \%$ & - & $0,00 \%$ & $\cdot$ & $0,00 \%$ & 4 & $0.45 \%$ & - & $0,00 \%$ & - & $0.00 \%$ \\
\hline LEXUS & 500 & $11,09 \%$ & 59 & $2,53 \%$ & 2 & $0.14 \%$ & 6 & $1.15 \%$ & 2 & $0.22 \%$ & 2 & $0,22 \%$ & - & $0,00 \%$ \\
\hline CHEVROLET & 711 & $15,77 \%$ & 328 & $14,08 \%$ & 3 & $0,21 \%$ & 1 & $0.19 \%$ & $\cdot$ & $0,00 \%$ & - & $0,00 \%$ & - & $0,00 \%$ \\
\hline MERCEDES BENZ & 86 & $1,91 \%$ & 78 & $3,35 \%$ & - & $0.00 \%$ & - & $0,00 \%$ & - & $0,00 \%$ & - & $0,00 \%$ & - & $0.00 \%$ \\
\hline GMC & 53 & $1,18 \%$ & 13 & $0,56 \%$ & 3 & $0,21 \%$ & - & $0.00 \%$ & $\cdot$ & $0.00 \%$ & - & $0.00 \%$ & - & $0.00 \%$ \\
\hline OTRAS & 55 & $1,22 \%$ & 68 & $2,92 \%$ & 7 & $0,50 \%$ & 63 & $12.09 \%$ & - & $0.00 \%$ & - & $0.00 \%$ & - & $0.00 \%$ \\
\hline TOTAL & 4509 & $100,00 \%$ & 2330 & $100,00 \%$ & 1400 & $100,00 \%$ & 521 & $100,00 \%$ & 892 & $100,00 \%$ & 909 & $100,00 \%$ & 991 & $100,00 \%$ \\
\hline
\end{tabular}

Con estos datos se demuestra que el mejor momento para la empresa surcoreana fue en el año 2015 donde alcanzó la cúspide con el 78.88\% de ventas anuales con el modelo como Sonata Hybrid Full, al cual un año más tarde se sumó en modelo Ioniq. 


\section{Fundamento Teórico}

\section{Hyundai Sonata Híbrido}

La marca Hyundai busca vehículos más eficientes con menor consumo de combustible generando así menor contaminación ambiental, introduciendo en el país autos de esta gama, con el modelo Sonata Hybrid, un vehículo tipo sedán de cuatro puertas, con una batería de alta tensión que se cuida sola y se autocarga con la electricidad generada cuando se cambia a modo híbrido cuando esta lo requiera, la batería alimenta a los motores eléctricos y duran de por vida según la gran marca comercial. Cuenta con un diseño aerodinámico que soportan a un poderoso motor interno a gasolina para mayor potencia y un eficiente motor eléctrico para una conducción más suave y amigable con el ecosistema. (Hyundai, 2018)

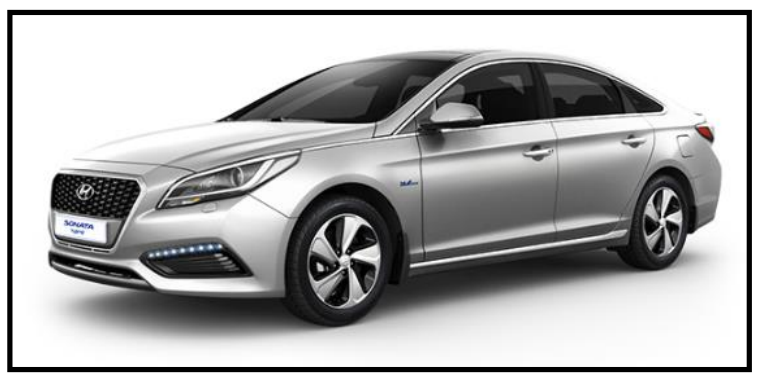

Figura 1. Hyundai Sonata Híbrido (Hyundai, 2018)

\section{Componentes del Hyundai Sonata Híbrido}

El Hyundai Sonata Híbrido tiene configuración en paralelo que ha sido desarrollado por Hyundai Motor Company, los embragues del motor de tracción y del motor híbrido son instalados justo entre ambos, esto permite activar el modo EV, incrementando la eficiencia del combustible y asegurando una excelente potencia y desempeño. (Hyundai, 2018).

El sistema que posee Hyundai posee una capacidad de ponerse en marcha sin ayuda del motor de combustión interna, con la ayuda del "Hybrid Started Generator" el motor de combustión interna se iguala en revoluciones, de esta manera el embrague se puede acoplar para que el usuario no sienta ningún tipo de movimiento brusco y que el vehículo desarrolle de una manera óptima.

Cuando el usuario decrece la velocidad el motor de combustión interna se desvincula y el generador empieza a recargar las baterías gracias a la inercia del automóvil y el movimiento de los neumáticos. 


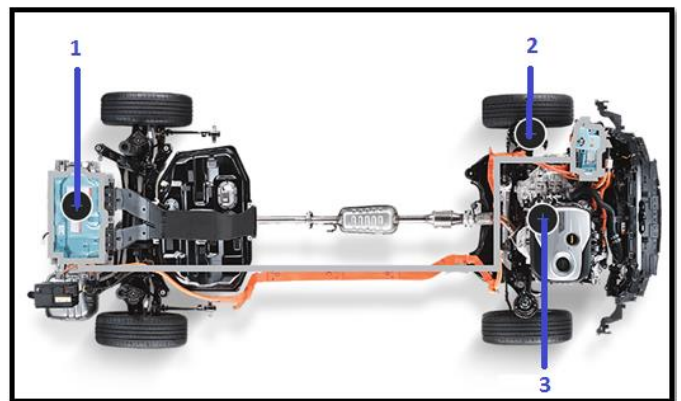

Figura 2. Ubicación de los componentes generales del sistema híbrido (Toyota, 2008)

Tabla 2. Descripción de los componentes generales del Sonata hibrido

\begin{tabular}{cl}
\hline Componente & Descripción \\
\hline $\mathbf{1}$ & Batería de Litio - Polímero \\
$\mathbf{2}$ & Sistema de frenado regenerativo \\
$\mathbf{3}$ & Motor Híbrido \\
\hline & (Autores, 2018)
\end{tabular}

En la siguiente figura se muestran además componentes como el Generador de arranque híbrido, cable de potencia y la transmisión de cambio.

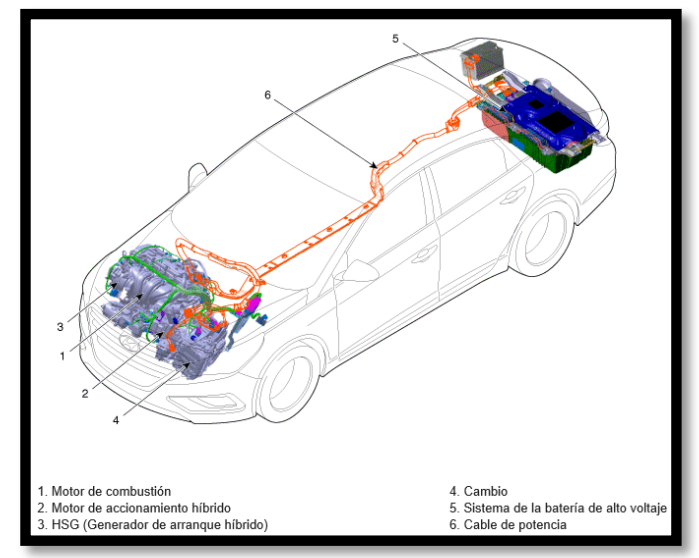

Figura 3. Ubicación de los componentes generales del sistema híbrido

(Hyundai, 2018)

\section{Materiales y Métodos}

\section{Batería de alta tensión del Hyundai Sonata Híbrido}

Las baterías híbridas para automóvil consisten en muchas celdas conectadas en serie y empacadas en un contenedor. Estos se llaman módulos, monitoreado diversos puntos importantes tales como la refrigeración, la calefacción, el voltaje, amperaje, la detección de fugas de alto voltaje, además de incluir los relés para garantizar una vida larga y segura.

Con más de una década de presencia en el mercado la batería de polímero de litio del Sonata Híbrido ha demostrado ser más que segura, ligera y duradera. (Hyundai, 2018) 


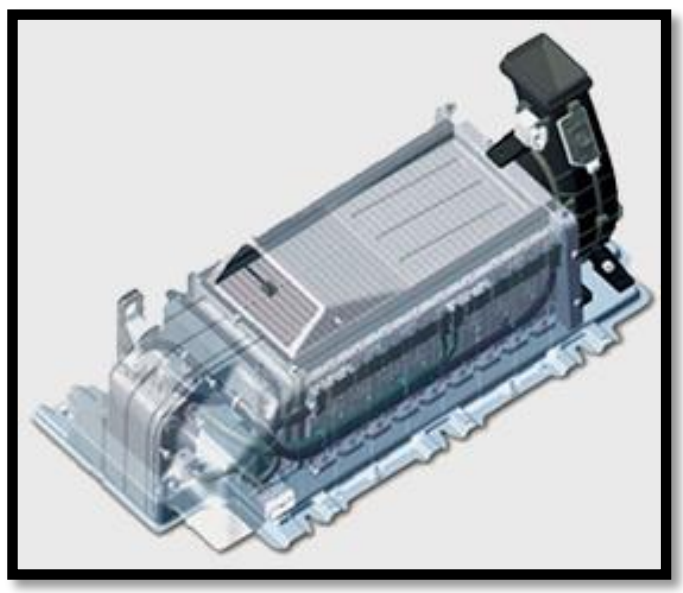

Figura 4. Paquete de baterías de polímero de litio del Hyundai Sonata (Hyundai)

Tabla 3. Datos generales del paquete de alta tensión (Hyundai, 2018)

\begin{tabular}{lc}
\hline \multicolumn{2}{c}{ Paquete de batería de alta tensión } \\
\hline Voltaje del paquete de baterías & $270 \mathrm{~V}$ \\
Número de células & 8 células x 9 módulos \\
Voltaje por cada célula & $3.75 \mathrm{~V}$ \\
\hline
\end{tabular}

\section{Charger Research}

El banco de trabajo "Charger Research" es un equipo que permite evaluar el estado, funcionamiento y también permite generar cargas y descargas de las baterías que vayamos a conectar. Las baterías que puede evaluar son baterías de carros híbridos y eléctricos; la unidad está en posibilidades de reparar las celdas internas de la batería.

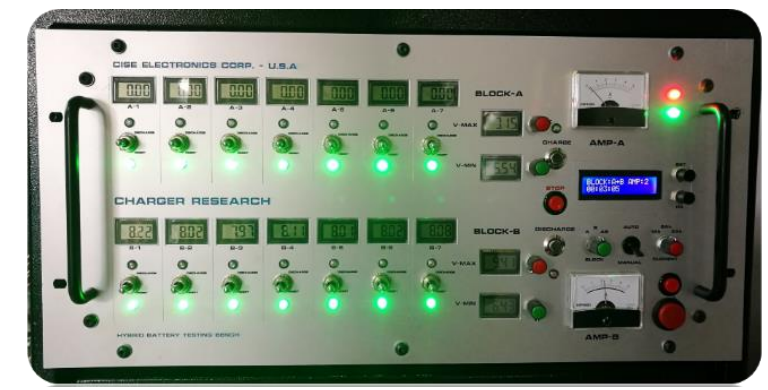

Figura 5. Panel de control del Banco de Pruebas para baterías híbridas (TAAET Electronics , 2017)

\section{Función}

El banco de pruebas Charger Research por motivos de seguridad tiene dos bloques por separados nombrados como bloque A y B. Por cada uno de los bloques se pueden conectar hasta siete celdas de baterías con una tensión nominal de $7.2 \mathrm{~V}$, el voltaje total no supera el valor de sesenta voltios para que no implique ningún daño para el usuario por exceso de voltaje 
De esta forma con los dos bloques se puede evaluar hasta 14 celdas al mismo momento. En cada celda que sea analizar, cargar o descargar posee un medidor individual de voltaje. Cada recuperación conlleva a una carga y descarga para cada celda con esto se analiza la corriente y tensión por medio de dos medidores para verificar los voltajes mínimos como el máximo de cada celda dependiendo a los voltajes que estén configurados en el equipo para descarga y carga de cada celda; cada bloque se configura con dos celdas por bloque. Cada bloque opera por separado el A o el B trabajan por separados.

\section{Partes}

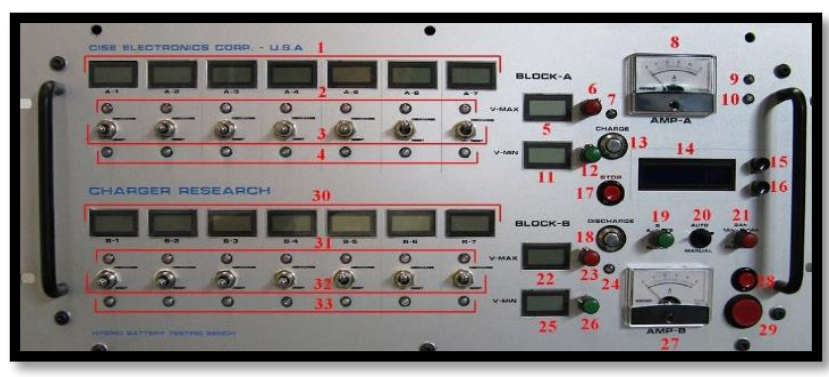

Figura 6. Partes del banco comprobador de baterías hibridas (TAAET Electronics , 2017)

El banco de pruebas para baterías híbridas consta de 33 partes que se describen a continuación:

Tabla 4. Partes del Charger Research

\begin{tabular}{|c|c|}
\hline \multicolumn{2}{|r|}{ Partes del Charger Research } \\
\hline 1 & Conjunto voltímetros bloque $\mathrm{A}$ \\
\hline 2 & Conjunto leds indicación voltaje fuera de rango alto bloque $\mathrm{A}$ \\
\hline 3 & $\begin{array}{l}\text { Conjunto llaves tres posiciones - Alta: forzar carga - Media: Posición normal de trabajo - Baja: Reseteo } \\
\text { - bloque A }\end{array}$ \\
\hline 4 & Conjunto leds indicación voltaje fuera de rango seteado bajo bloque A \\
\hline 5 & Voltímetro indicación máximo voltaje de carga admitido bloque A \\
\hline 6 & Potenciómetro selección máximo voltaje de carga bloque A \\
\hline 7 & Led indicación \\
\hline 8 & Amperímetro para carga y descarga bloque A \\
\hline 9 & Led indicación \\
\hline 10 & Led indicación \\
\hline 11 & Voltímetro indicación mínimo voltaje de descarga admitido bloque A \\
\hline 12 & Potenciómetro selección mínimo voltaje de descarga bloque A \\
\hline 13 & Pulsador comienzo de carga bloque A \\
\hline 14 & Display seteos bloque A y B \\
\hline 15 & Pulsador visualización de seteos - selección de bloque - corriente - tiempo de ambos bloques \\
\hline 16 & Pulsador aprobación seteos \\
\hline 17 & Pulsador detención de actividad \\
\hline 18 & Pulsador comienzo de descarga \\
\hline 19 & Selector de bloques $\mathrm{A}-\mathrm{B}$ o ambos \\
\hline 20 & Selector automático/manual \\
\hline 21 & Selector corriente (amp) para carga y descarga \\
\hline 22 & Voltímetro indicación máximo voltaje de carga admitido bloque B \\
\hline 23 & Potenciómetro selección máximo voltaje de carga bloque B \\
\hline 24 & Led indicación \\
\hline 25 & Voltímetro indicación mínimo voltaje de carga admitido bloque B \\
\hline
\end{tabular}




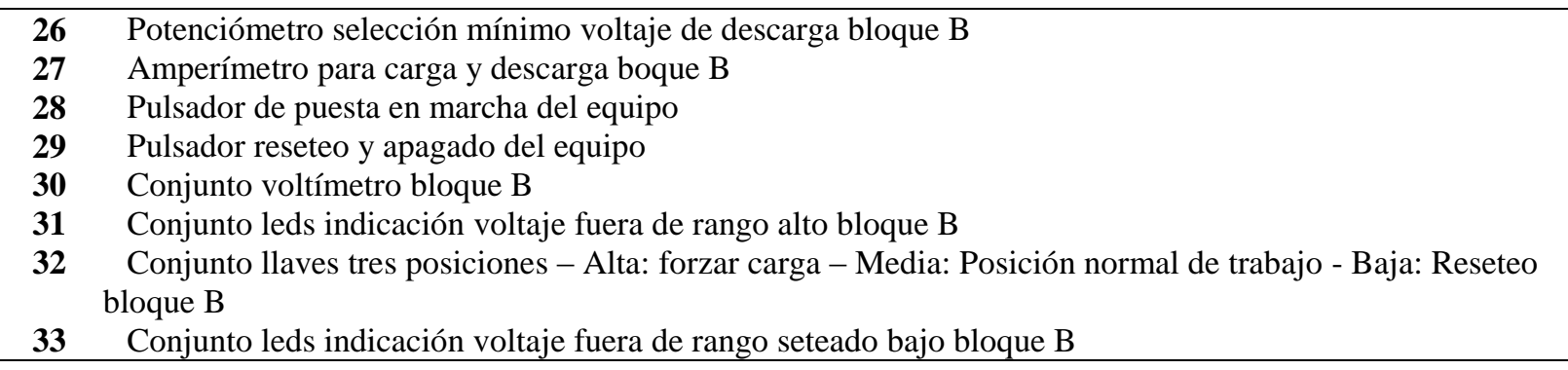

\section{Pruebas}

Si nota que su híbrido tiene menos energía que antes, que no responde como solía hacerlo, las capacidades de su batería pueden estar bajo algún tipo de falla o deterioro ya que necesita que la batería esté en óptimas condiciones para transferir toda esa energía a la tracción eléctrica. Si su paquete de batería no proporciona la cantidad adecuada de energía sentirá el vehículo más pesado o lento.

Por lo que la recuperación de baterías de alta tensión está ganando mucha popularidad y los propietarios de vehículos híbridos pueden disfrutar de inmediato del rendimiento de su unidad nuevamente. El proceso de recuperación de la batería híbrida es sencillo, aunque largo, cuesta una fracción del costo de una batería nueva. Para hacer una diferencia en el medio ambiente, como ha sido el propósito de la mayoría de los conductores de vehículos híbridos, ahora la recuperación de la batería híbrida puede ayudar a eliminar los desechos en los basureros.

La batería del Hyundai Sonata Híbrido, por ejemplo, consta de 72 celdas individuales y cuando hay un problema con la batería, lo más probable es que se deba a una de las celdas, no a todas. Cada una de las 72 celdas tiene su propio estado de salud y en un momento dado pueden estar en un estado diferente. Con el tiempo, los módulos en peor estado de este paquete terminan activando un código de falla y eso a veces puede obligar a un distribuidor o un taller a reemplazar la batería completa. Sin embargo, es posible que uno o dos de los módulos deban reemplazarse y que no todo el paquete o la batería este en mal estado. El proceso de recuperación significa que el paquete se recicla extrayendo y reemplazando las celdas en mal estado. Híbrido.

En la siguiente figura se muestra la configuración de la batería de alta tensión del Sonata

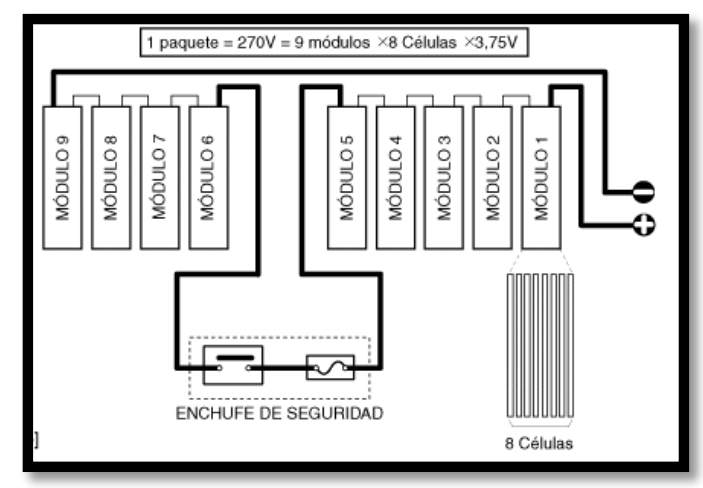

Figura 7. Partes del banco comprobador de baterías hibridas (Hyundai, 2018) 


\section{Proceso de Recuperación de las baterías de alta tensión}

\section{Diagnóstico Inicial}

El diagnóstico inicia cuando encontramos algún código de falla en el tablero que indique error o avería en el sistema de alta tensión, lo cual probablemente podría ser la batería. Para verificar se inicia el proceso con ayuda de un escáner, como el GDS. No olvide ingresar los datos correctos del automóvil para asegurar una correcta conexión.

\section{Desmontaje de la Batería}

Luego verificar con el escáner la existencia de un código de falla referente a la batería se procede a desmontar la batería de alta tensión que usualmente se encuentra ubicada por debajo del asiento posterior, para esto no olvide tomar las precauciones con el circuito de alto voltaje para evitar futuros contratiempos.

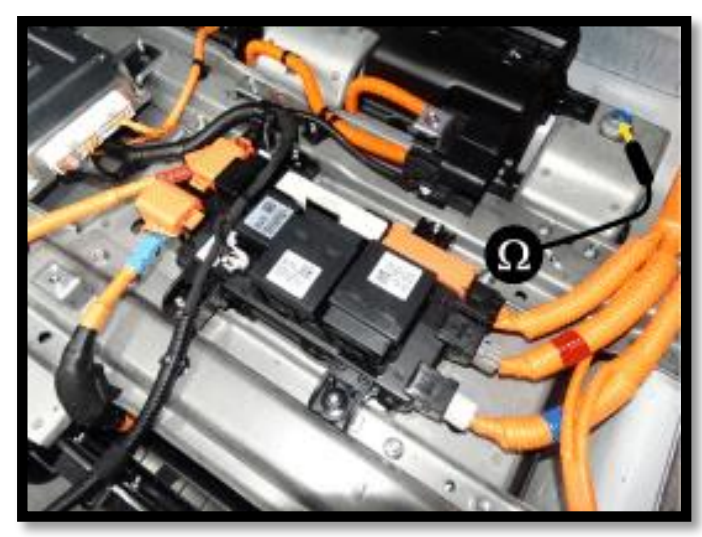

Figura 8. Conjunto de Batería Sonata Híbrido (Hyundai, 2018)

\section{Proceso de descarga de la batería}

1) Armamos dos bloques de 7 celdas cada uno ya que el equipo que utilizamos solo permite hasta un máximo de 14 celdas.

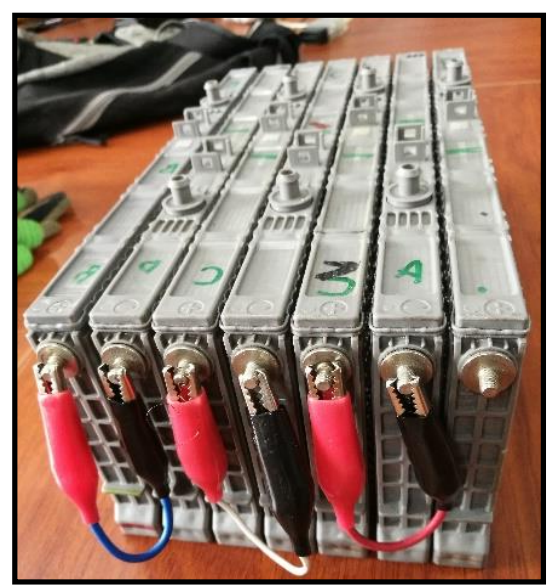

Figura 9. Conexión en serie de celdas- (Autores, 2018) 
2) Realizamos la conexión a través de los terminales del banco hacia cada una de las celdas

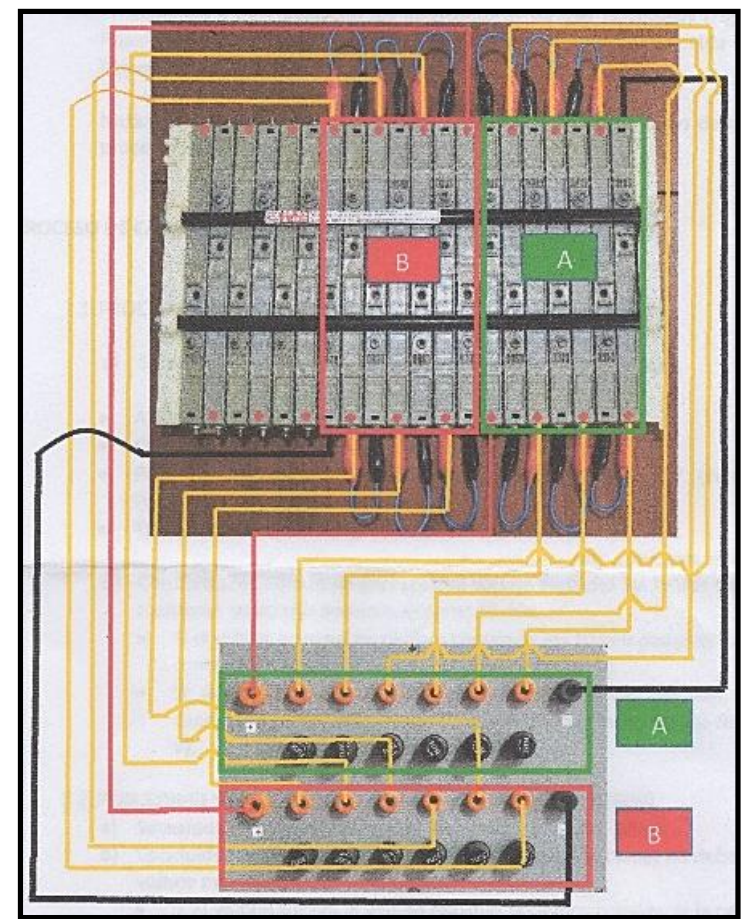

Figura 10. Diagrama de conexión

(TAAET Electronics , 2017)

3) Seteamos en el panel de control del banco

$\checkmark$ Selecciona automático (\#20 todos los componentes)

$\checkmark$ Selecciona corriente de descarga 1 amp (\#21 todos los componentes)

$\checkmark$ Selecciona el voltaje mínimo con el potenciómetro de selección (\#12 y \#26 todos los componentes)

$\checkmark$ Selecciona el tiempo de 2 horas de descarga (\#15 todos los componentes)

Nota: el sistema de descarga automática se detendrá al momento que cualquiera de sus celdas llega al mínimo seleccionado de 6 voltios.

4) Procedemos a descargar con los interruptores de tres posiciones (\#3 y \#32 todos los componentes) y descargamos cada celda hasta que llegue a 5 voltios

Proceso de carga de la batería

Proceso de precarga

1) Programamos en el panel de control del banco 30 min - 1 Amp. 
Selecciona automático (\#20 todos los componentes)

$\checkmark$ Selecciona corriente de carga 1 Amp

$\checkmark$ Selecciona el máximo de voltaje en 9 v con el potenciómetro (\#6 y \#23 todos los componentes)

$\checkmark$ Selecciona el tiempo de 30 min (\#15 todos los componentes)

2) Concluido los 30 min de precarga, el voltaje en todas las celdas tendría que mantener su voltaje nominal. Esperar durante 10 minutos.

$\checkmark$ Si el voltaje nominal no cambia dentro de los 10 minutos podemos consideras como celda aprobada en el primer procedimiento.

$\checkmark$ Si el voltaje nominal disminuye transcurriendo este tiempo podemos consideras como celda sospechosa para el cambio.

Procedimiento de carga a una capacidad del $30 \%$

1) Programamos en el panel de control del banco 60 min - 2 Amp.

2) Concluido los 60 min de carga, el voltaje en todas las celdas no debe pasar el voltaje máximo.

3) Si el voltaje supera el voltaje máximo podemos considerar la celda como sospechosa para el cambio.

Proceso de descarga

1) Configuramos en el panel de control una descarga a 1 Amp durante 2 horas

2) Mientras las celdas se están descargando las iremos categorizando tomando en cuenta el tiempo en que les toma llegar a su voltaje mínimo. Guiándonos por la siguiente tabla.

Tabla 5 Categorización de las celdas

\begin{tabular}{cc}
\hline Categoría & Tiempo (horas) \\
\hline A & $1: 41-2: 00$ \\
B & $1: 26-1: 40$ \\
C & $1: 10-1: 25$ \\
Celda defectuosa & Menos de $1: 10$
\end{tabular}

(TAAET Electronics , 2017)

\section{Resultados y Discusión}

Luego de seguir el proceso de recuperación se obtuvo que una de las 72 celdas que posee el Hyundai Sonata presentaba falla, que según la categorización estaba defectuosa, lo que no 
permitía el correcto funcionamiento del vehículo. Considerando que el GDS nos dio el código de falla "POA7F".

Tabla 6. Categorización de las celdas obtenidas en la prueba (Autores)

\begin{tabular}{cc}
\hline MODULO & TIEMPO EN HORAS \\
\hline MODULO 1 & $1: 00$ \\
MODULO 2 & $2: 00$ \\
MODULO 3 & $1: 58$ \\
MODULO 4 & $1: 57$ \\
MODULO 5 & $2: 00$ \\
MODULO 6 & $1: 59$ \\
MODULO 7 & $2: 00$ \\
MODULO 8 & $1: 59$ \\
MODULO 9 & $2: 00$ \\
\hline
\end{tabular}

Siendo el módulo 1 el que presenta fallas según el tiempo de descarga se las categoriza tipo defectuosa, mientras las demás estaban en categoría A por lo que solo es necesario el cambio de esas celdas por otras de categoría A para volver a poner en buen funcionamiento el vehículo. 


\section{Conclusiones}

Tras el análisis con los respectivos instrumentos de comprobación, scanners y banco de prueba se puede lograr conocer el estado respectivo de cada celda del pack de baterías del vehículo Hyundai Sonata Híbrido, de esta manera se puede reemplazar las celdas según la clasificación que tenga las celdas que se están evaluando, ya sean tipo "A", "B", "C" o "X", de esta manera se puede obtener un pack de baterías balanceado con las celdas de las categorías correspondientes, de este modo el vehículo puede circular correctamente sin ningún tipo de problemas o DTC que se haya presentado por desperfecto en el pack de baterías del circuito de alto voltaje.

Viendo el crecimiento considerable de este mercado desde su aparición, el enfoque de los talleres hacia el mantenimiento y reparación de esta tecnología es completamente necesario ya que sin duda invadirá nuestro mercado con el pasar de los años. Por lo que la necesidad de especializarse y aprender de estos procedimientos permitirá obtener otro mercado.

Sin duda alguna el proceso de recuperación de batería es lo más conveniente al momento de considerar el gasto que representa reemplazar la batería completa, sin dejar de lado los beneficios medioambientales que representa reusar la batería que ya posee nuestro vehículo y solo reemplazar las celdas en mal estado.

El vehículo cuenta con diversos sistemas de seguridad además de la protección que el equipo personal debe tener, por lo que seguirlos sin evadir paso alguno es esencial siendo consiente de los altos voltajes que este sistema maneja. 


\section{Bibliografía}

AEADE. (2017). Asociacion De Empresas Automotrices Del Ecuador. Obtenido de http://www.aeade.net/anuario-2016/anuario2016.pdf

Cars. (25 de 06 de 2014). Cars. Obtenido de https://www.cars.com/research/toyotahighlander_hybrid-2014/

Comercio, R. L.-E. (2013). Revista Líderes. Obtenido de http://www.revistalideres.ec/lideres/hyundai-le-apuesta-hibrido-pais.html

Curtis Anderson, J. A. (2010). Electric and Hybrid Cars. Mcfarland.

Ecolectric Battery - (23 de 05 de 2017). Ecolectric Battery . Obtenido de http://ecolectricbattery.com/1/post/2017/05/modelos-de-vehiculos-hibridos-guia.html

Hybrid Automotive. (2016). Hybrid Automotive. Obtenido de https://hybridautomotive.com/pages/install-hh1

Hyundai. (s.f.). Costa Rica.

Hyundai. (2018). Hyundai. Obtenido de https://www.hyundai.com.ec/showroom/sonata-hybrid/

Hyundai. (2018). Manual de Servicio del Sonata . Hyundai.

Liker, J. K. (2006). Japanese automakers, US suppliers and supply chain superiority. Supply Chains and Total Product Systems: A Reader.

Motor trend. (01 de 03 de 2008). Motor trend. Obtenido de http://www.motortrend.com/cars/toyota/highlander/2008/

Pèrez, J. (14 de 02 de 2017). Motor Zeta. Obtenido de https://www.elperiodico.com/es/motor/quienes-somos.shtml

Sánchez, M. \&. (2017). Análisis, diagnóstico y propuesta de mejora para una cadena de suministro de autopartes en el Perú utilizando el modelo SCOR.

TAAET Electronics . (2017). Manual de uso del banco de pruebas de bateria hibrida. Guayaquil.

Toyota. (2008). Emergency response guide. Toyota motor company.

Toyota. (2015). Blog Toyota. Obtenido de http://blog.toyota.co.uk/how-does-toyota-hybridsynergy-drive-work

Toyota. (2018). Toyota de Puerto Rico, Corp. Obtenido de http://www.toyotapr.com/hibridos 